\title{
Spectro-polarimetric observation of the fine structure of a quiescent filament
}

\author{
W. G. Zong ${ }^{1}$, Y. H. Tang ${ }^{1}$, C. Fang ${ }^{1}$, P. Mein ${ }^{2}$, N. Mein ${ }^{2}$, and A. A. Xu ${ }^{1}$ \\ 1 Department of Astronomy, Nanjing University, 210093, Nanjing, PR China \\ 2 Observatoire de Paris, Section de Meudon, 92195, Meudon, France
}

Received 24 July 2003 / Accepted 21 August 2003

\begin{abstract}
This paper presents the spectro-polarimetric measurements of a big quiescent filament observed by the MSDP mode of the THEMIS on August 24, 2000. The $\mathrm{H} \alpha$, CaII 8542 and NaI D2 line profiles of a segment of the filament were obtained. By use of the $\mathrm{H} \alpha$ images with high spatial resolution, the two barb endpoints were identified. The parameters at the barbs' endpoints, including intensity, velocity and longitudinal magnetic field were measured. Using the data with high spatial resolution ( $0.16^{\prime \prime}$ per pixel), we have found the following results. 1) There was mass motion at the barb endpoints in the chromosphere, the values and the directions of the mass motion at the barb endpoints change in several minutes. 2) The two barb endpoints are located between the majority polarities and the minority polarities.
\end{abstract}

Key words. line: profiles - Sun: filaments - Sun: magnetic fields

\section{Introduction}

As is well known, filaments or prominences have fine structures that branch out from their main body and reach down to the chromosphere. These structures are called barbs. Martin (1998a) considered barbs as magnetic conduits along which mass is continuously guided and transported to and from the chromosphere; the barb endpoints of filaments are the locations where the filaments anchor to the chromosphere.

The relationship of the endpoints of filament barbs to photospheric magnetic fields has long been a puzzle. Early studies showed that there is an association between the barbs and the boundaries of supergranules, implying a relationship with network magnetic fields (Ploceniak \& Rompolt 1973). However, recently Engvold (1998) found an anti-correlation between the barb endpoints and network magnetic fields. They illustrated the termination of some barb endpoints within cells in the absence of ephemeral regions. This led to the impression that some barb endpoints are located in extremely weak photospheric magnetic fields. However, Martin \& Echols (1994) identified some barbs that terminated in positive or negative poles of ephemeral regions. They found that the barbs are related to patches of minority polarity on each side of a filament and the minority polarity was defined as that opposite to the background one of the network on the same side of the filament. An interesting interpretation of filament barbs and their relation to minority-polarity fields has been presented by Aulanier et al. (1998). They assumed a basic magnetic

Send offprint requests to: Y. H. Tang, e-mail: yhtang@nju.edu.cn configuration consisting of a twisted flux tube whose axis coincides with the large-scale photospheric neutral line. Wherever this imposed background field overlies pockets of minoritypolarity flux, the field lines sag downward to form dips, inside which the prominence material is supported. Wang (2001) found in the HeII $\lambda 304 \AA$ line the barbs extended from the main body of the filament to neutral lines, where the oppositepolarity flux elements were contiguous to each other.

In this paper the study of a big quiescent filament observed by THEMIS is presented. Two dimensional spectropolarimetric observation of the barb endpoints of the filament was made with high spatial resolution. The line profiles and the parameters at the barb endpoints, including intensity, velocity and longitudinal magnetic field, were obtained.

\section{Spectro-polarimetric observations with THEMIS/MSDP}

THEMIS is a $90 \mathrm{~cm}$ aperture telescope, especially designed for observations with high spatial resolution and polarization measurements (For more details on the THEMIS capabilities see http: //www. themis.iac.es).

On August 24, 2000 a big quiescent filament was seen in $\mathrm{H} \alpha$ image on the solar disk. It was located in the northern hemisphere near the center of the solar disk (W15 N30) and looked like a shrimp. Spectro-polarimetric observations of the filament in $\mathrm{H} \alpha$, CaII 8542 and NaI D2 5890 lines were carried out with the THEMIS telescope, operating in the MSDP mode (Mein 2002). The seeing was good and was estimated to be better than $1^{\prime \prime}$ during the observation. 

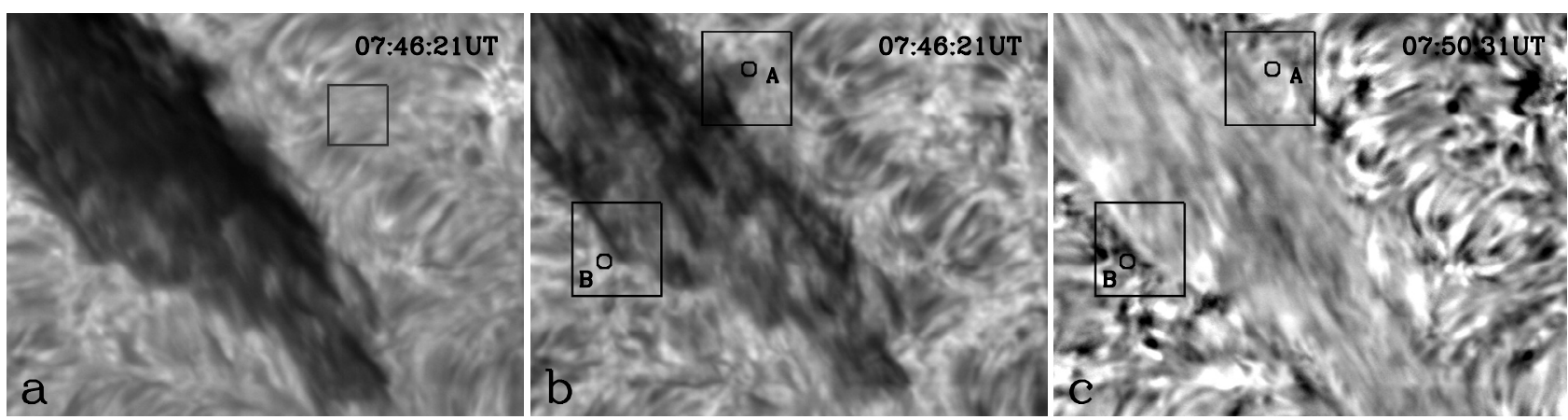

Fig. 1. a) Segment of the quiescent filament at the center of $\mathrm{H} \alpha$ line $(6563 \AA)$. The region in the black rectangle was selected as the quiet background $\left(13^{\prime \prime} \times 13^{\prime \prime}\right)$ for calibration. b) The segment at $\Delta \lambda=-0.315 \AA$ from the $\mathrm{H} \alpha$ line center. Two barbs were located in the black rectangles and marked with "A" and "B". The black circles indicate the locations of their endpoints. c) Velocity field obtained at $\pm 0.18 \AA$ of $\mathrm{H} \alpha$ lines by using bisector method. The mass flows up in the white areas and down in the black areas. The barbs were marked the same as in Fig. 1b. The images were recorded on August 24, 2000. East is to the left and north is up.
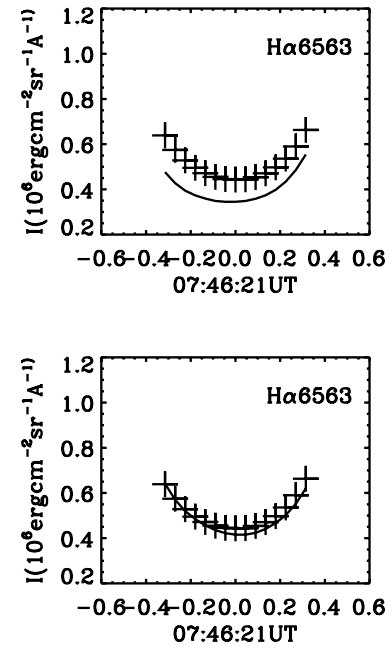

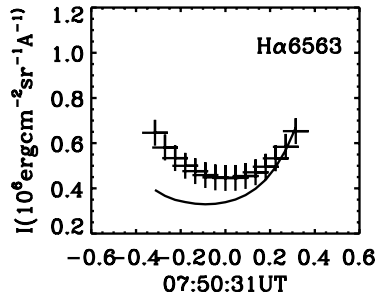

a: the profiles of the barb A's endpoint

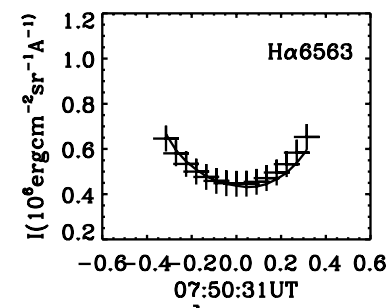

$\mathrm{b}:$
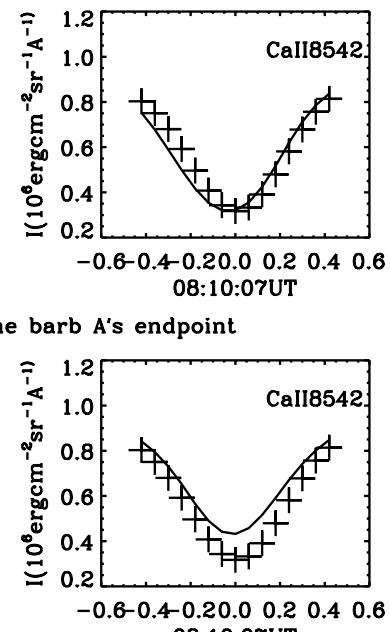

08:10:07UT

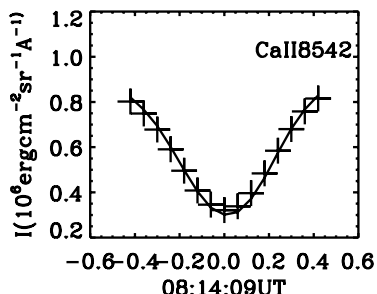

08:14:09UT

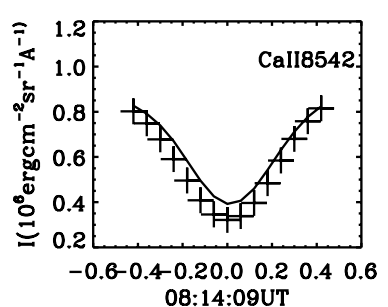

08:14:09UT

Fig. 2. Line profiles of the two barb endpoint (solid lines), obtained by averaging the profiles at $7 \times 7\left(1.1^{\prime \prime} \times 1.1^{\prime \prime}\right)$ pixels around the center pixels of the black circles in Fig. 1b. The lines marked by "+" are the mean line profiles of a quiescent background (an average over 13" $\left.\times 13^{\prime \prime}\right)$ in the black rectangle in Fig. 1a.

The distance between successive MSDP channels is $90 \mathrm{~m} \AA$ and $120 \mathrm{~m} \AA$ for the case of $\mathrm{H} \alpha$ and CaII $8542 \AA$ respectively. Using the code provided by Mein to deal with the raw data, we obtained interpolated $\mathrm{H} \alpha$ and CaII $8542 \AA$ images both at 15 wavelength values. The distances between wavelengths are $45 \mathrm{~m} \AA$ and $60 \mathrm{~m} \AA$ respectively. From the images, the 2D spectra were obtained. The scans covered a field of view of $697 \times 542$ pixels $\left(112^{\prime \prime} \times 87^{\prime \prime}\right)$, and were made twice successively for the $\mathrm{H} \alpha$ and CaII lines; the $\mathrm{H} \alpha$ line observations started at 07:46:21 UT and 07:50:31 UT, while the CaII lines were recorded at 08:10:07 UT and 08:14:09 UT. The scan for NaI D2 line was carried out only once, but all Stokes parameters were recorded, allowing us to determine the photospheric magnetic field of the region. The longitudinal magnetic field $B_{\|}$ was derived from Stokes $V$ only.

\section{Data analysis}

Figures 1a and $\mathrm{b}$ show a segment of the filament observed by THEMIS. Figure 1a was obtained at the $\mathrm{H} \alpha$ line center, and the filament can be seen clearly. Figure $1 \mathrm{~b}$ was recorded at $\Delta \lambda=-0.315 \AA$ from the $\mathrm{H} \alpha$ line center, on which we can see two barbs marked with "A" and "B" in the black rectangles. Comparing all the images at 15 wavelength channels in the $\mathrm{H} \alpha$ line, we found that the barbs extend away farthest from the filament spine shown in Fig. 1b, which was then used to identify the barb endpoints. From the image, we can see that the barbs are right-bearing, as expected for northern hemisphere filaments. This is consistent with the empirical chirality rule proposed by Martin (1998). Figure 1c presents the $\mathrm{H} \alpha$ velocity field, which was obtained at $\pm 0.18 \AA$ by using the bisector method. The mass flows up in the white areas and down in the black areas. The barbs are indicated as in Fig. $1 b$.

Figure 2 shows the profiles of the two barbs' endpoints in the $\mathrm{H} \alpha$ and CaII lines. The mean profiles of the quiet background (an average over $13^{\prime \prime} \times 13^{\prime \prime}$ ) are marked by "+" and the solid lines are profiles of the barbs' endpoints. Using the bisector method, we obtained the velocity at the barbs' endpoints. We calculated the velocities in $\mathrm{H} \alpha$ channels at $\pm 0.18 \AA$ from the line center at 07:46:21 UT and 07:50:31 UT. 

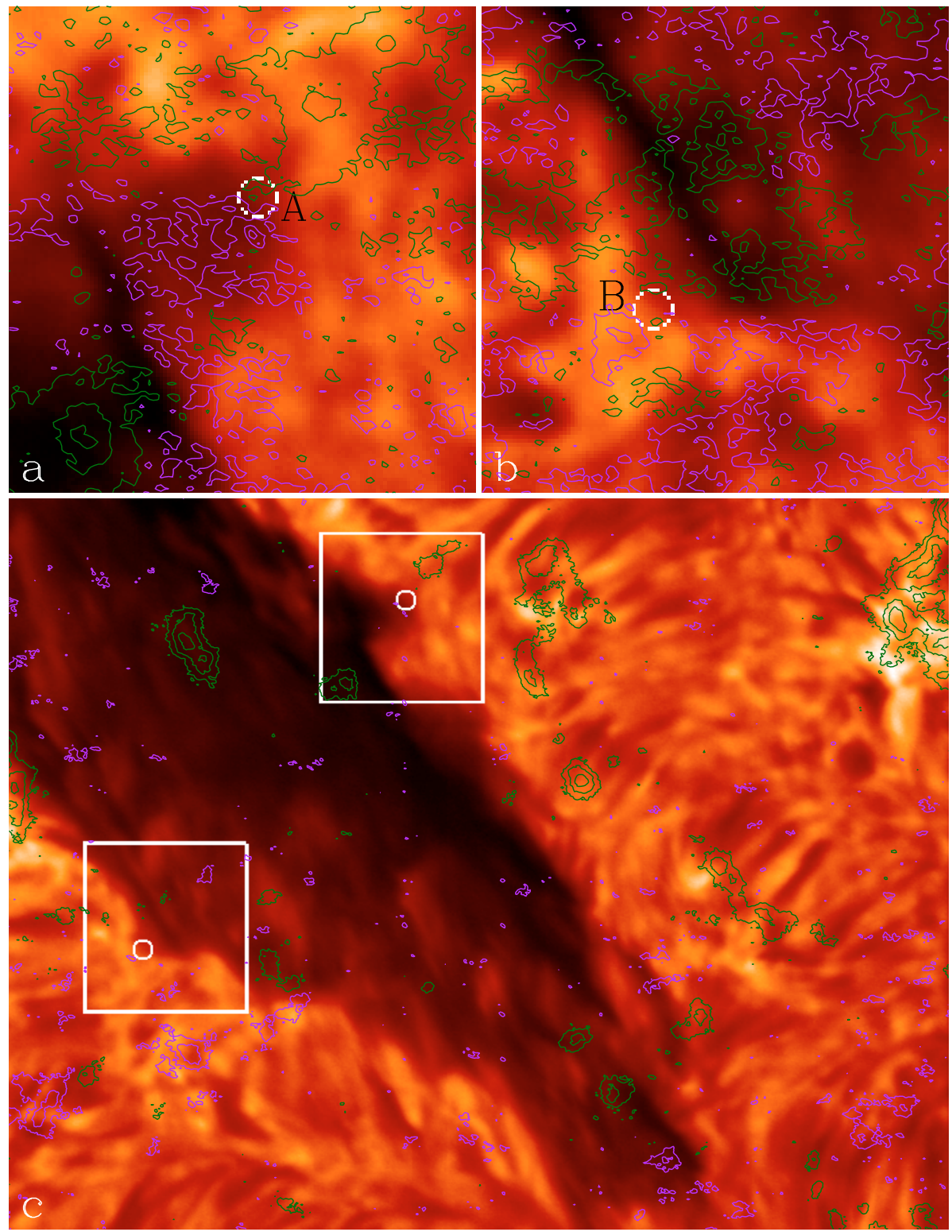

Fig. 3. Co-alignments of intensity fields and the longitudinal photospheric magnetic field, which was obtained from the NaI $5890 \AA$ line at 08:34:04 UT. The directions of magnetic fields are down in the green contours and up in the purple contours. a)-b) The longitudinal magnetic field contour on the $\mathrm{H} \alpha$ barbs images (at $\Delta \lambda=-0.315 \AA$ ). The sizes of the regions are $120 \times 125$ pixels $\left(19^{\prime \prime} \times 20^{\prime \prime}\right)$, which are the same regions corresponding to the black rectangles in Fig. $1 \mathrm{~b}$ and the white rectangles in Fig. 3c. The barb endpoints were identified by white circles. c) The overlap of the longitudinal magnetic field contour on the $\mathrm{H} \alpha$ image at line center of our observed field. The levels in green lines are $-21 \mathrm{G}$, $-93 \mathrm{G},-166 \mathrm{G}$ in a), $-21 \mathrm{G},-52 \mathrm{G}$ in b) and $-62 \mathrm{G},-145 \mathrm{G},-270 \mathrm{G}$ in c). The levels in purple lines are $17 \mathrm{G}, 52 \mathrm{G}$ in a) $-\mathbf{b})$ and $52 \mathrm{G}, 135 \mathrm{G}$ in c). East is to the left and north is up.

The velocities in CaII channels were measured at $\pm 0.24 \AA$ from the line center at 08:10:07 UT and 08:14:08 UT. The velocities at the two barbs' endpoints are presented in Table 1. The values are the averages over $7 \times 7$ pixels $\left(1.1^{\prime \prime} \times 1.1^{\prime \prime}\right)$. We also averaged the velocities over $11 \times 11$ pixels $\left(1.7^{\prime \prime} \times 1.7^{\prime \prime}\right)$. The results change slightly, but the signs are the same. It must be noted that, for absorbing features and unreversed profiles, the signs of velocities are correct, but the amplitude of the Doppler shift may be underestimated, so the real values of the velocities at a barb endpoint may be greater than the values presented in Table 1. This means that the mass at the barb endpoint flows up or down at high speed. Moreover, we found that the values and
Table 1. The velocities of the barb endpoint (Unit:m/s). Positive velocities correspond to redshifts.

\begin{tabular}{|c|c|c|c|c|}
\hline \hline line & \multicolumn{2}{|c|}{$\mathrm{H} \alpha$} & \multicolumn{2}{c|}{ CaII } \\
\hline$t(\mathrm{UT})$ & $07: 46: 21$ & $07: 50: 31$ & $08: 10: 07$ & $08: 14: 08$ \\
\hline$v_{\mathrm{A}}$ & -600 & -2040 & -540 & 190 \\
$v_{\mathrm{B}}$ & 280 & 760 & -130 & 240 \\
\hline
\end{tabular}

the directions of the velocities changed much both in the $\mathrm{H} \alpha$ and CaII lines in several minutes.

Figures $3 \mathrm{a}$ and $3 \mathrm{~b}$ show the photospheric longitudinal magnetic fields around the barb endpoints. In the images, the barb 


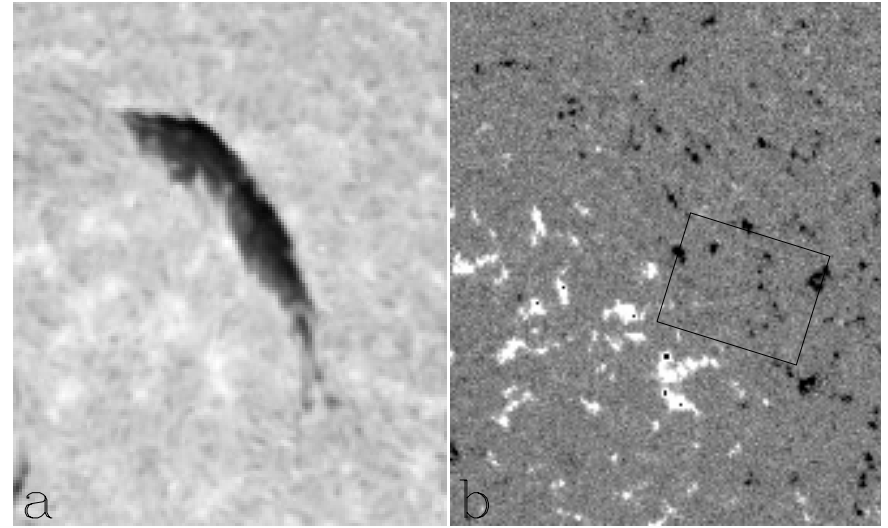

Fig. 4. a) $\mathrm{H} \alpha$ image of the filament observed at 06:44:00 UT at the Observatory of Paris. b) The longitudinal magnetic field around the filament obtained at 07:59:30 UT by SOHO/MDI. The region observed by us identified by black rectangle. The white areas are the regions with upward longitudinal magnetic field and the black ones are downward. We used the full disk $\mathrm{H} \alpha$ image and MDI image to co-align each other. The two images are for the same region on the solar disk. The size of the region is $320^{\prime \prime} \times 380^{\prime \prime}$. East is to the left and north is up.

endpoints were marked by the centers of the white circles. We found that they were located in the weak magnetic field regions between opposite polarities. Figure 4 depicts the $\mathrm{H} \alpha$ image of the full filament and the corresponding magnetic field from SOHO/MDI. As expected, we see the filament lies along the longitudinal field neutral line. The majority polarity is negative on the right to the filament spine and positive on the left. Figure $3 \mathrm{c}$ presents the magnetic field of our observed field. The majority polarities near the observed segment of the filament can be seen clearly. In Figs. $3 a$ and $b$ the minority polarities are also displayed clearly, although they are close to noise level. By comparing Figs. 3 and 4, we found the barb endpoints were located in the regions between the minority polarities and the majority polarities.

\section{Discussion and summary}

Using the $\mathrm{H} \alpha$ image obtained by the MSDP mode of the THEMIS with high spatial resolution, we have identified two barb endpoints. By use of spectro-polarimetric observation in
$\mathrm{H} \alpha$, CaII 8542 and NaI D2 lines, the parameters at the barb endpoints of a big quiescent filament, including intensities, velocities and longitudinal magnetic fields, have been measured and presented.

We found that the barbs terminated in the regions of very weak magnetic field. The barb endpoints were located between the majority polarities and the minority polarities. This result is in favor of the model proposed by Aulanier et al. (1998).

The line profiles of the barbs' endpoints indicate that there are notable mass motion in the barbs and the velocities change rapidly. However, at present, it is not clear whether the mass is transported from and to the spine along barbs. Nevertheless, it is obvious that the barbs are important factors for formation and development of filaments.

In our paper, the number of barbs is limited. Our conclusion thus is not complete. There are still many puzzles regarding their true nature. These puzzles should be resolved by studying more barbs with observations of high spatial and spectral resolution.

Acknowledgements. We would like to give our sincere thanks to Dr. G. Ceppatelli, Dr. C. Briand and other stuffs at the Spanish Observatorio del Teide of the Institute de Astrofisica de Canarias for their enthusiastic help during C.F. and Y.H.T's stay at the observatorio del Teide. This work was supported by NKBRSF (G20000783, G20000784) and the projects (No. 10073005, No. 49990451) from the National Natural Science Foundation of China.

\section{References}

Aulanier, G., Demoulin, P., Mein, N., et al. 1998, A\&A, 329, 1125

Engvold, O. 1998, in New perspectives on solar prominences, ed. D. Webb, D. M. Rust, \& B. Schmieder, ASP Conf. Ser., 150, 23

Martin, S. F., \& Echols, C. R. 1994, Solar Surface Magnetism, ed. R. J. Rutten \& C. J. Schrijver (Dordrecht, Holland: Kluwer Academic Publishers), 339

Martin, S. F. 1998a, Sol. Phys., 182, 107

Martin, S. F. 1998b, in New perspectives on solar prominences, ed.

D. Webb, D. M. Rust, \& B. Schmieder, ASP Conf. Ser., 150, 419 Mein, P. 2002, A\&A, 381, 271

Ploceniak, S., \& Rompolt, B. 1973, Sol. Phys., 29, 399

Wang, Y.-M. 2001, ApJ, 560, 456 